\title{
Structural and functional role of bases 32 and 33 in the anticodon loop of yeast mitochondrial tRNA
}

\author{
ARIANNA MONTANARI, ${ }^{1}$ CRISTINA DE LUCA, ${ }^{1}$ PATRIZIO DI MICCO ${ }^{2}$ VERONICA MOREA, ${ }^{3}$ \\ LAURA FRONTALI, ${ }^{1}$ and SILVIA FRANCISCI ${ }^{1,4}$ \\ ${ }^{1}$ Department of Biology and Biotechnology, Pasteur Institute, Cenci Bolognetti Foundation, Sapienza University of Rome, 00185 Rome, Italy \\ ${ }^{2}$ Department of Biochemical Sciences "A. Rossi Fanelli", Sapienza University of Rome, 00185 Rome, Italy \\ ${ }^{3} \mathrm{CNR}$ - National Research Council of Italy, Institute of Molecular Biology and Pathology, 00185 Rome, Italy
}

\begin{abstract}
Previous work has demonstrated the usefulness of the yeast model to investigate the molecular mechanisms underlying defects due to base substitutions in mitochondrial tRNA genes, and to identify suppressing molecules endowed with potential clinical relevance. The present paper extends these investigations to two human equivalent yeast mutations located at positions 32 and 33 in the anticodon loop of tRNA ${ }^{\text {lle }}$. Notwithstanding the proximity of the two $T>C$ base substitutions, the effects of these mutations have been found to be quite different in yeast, as they are in human. The T32C substitution has a very severe effect in yeast, consisting in a complete inhibition of growth on nonfermentable substrates. Conversely, respiratory defects caused by the T33C mutation could only be observed in a defined genetic context. Analyses of available sequences and selected tRNA threedimensional structures were performed to provide explanations for the different behavior of these adjacent mutations. Examination of the effects of previously identified suppressors demonstrated that overexpression of the TUF1 gene did not rescue the defective phenotypes determined by either mutation, possibly as a consequence of the lack of interactions between EF-Tu and the tRNA anticodon arm in known structures. On the contrary, both the cognate IleRS and the noncognate LeuRS and ValRS are endowed with suppressing activities toward both mutations. This allows us to extend to the tRNA ${ }^{\text {lle }}$ mutants the crosssuppression activity of aminoacyl-tRNA synthetases previously demonstrated for tRNA ${ }^{\text {Leu }}$ and tRNA ${ }^{\text {Val }}$ mutants.
\end{abstract}

Keywords: yeast; tRNA mutations; anticodon loop; mitochondrial diseases

\section{INTRODUCTION}

Saccharomyces cerevisiae is a very appropriate model organism to study the effect of mt mutations for several reasons. First, cells can shift to a fermentative metabolism in case of respiratory defects; therefore, the underlying mechanisms can be investigated in cells growing on fermentative carbon sources. Further, yeast mt DNA can be manipulated via biolistic transformation, which allows site-specific DNA substitutions to be introduced into the $\mathrm{mt}$ genome, following in vitro modification of the DNA. We have previously

Abbreviations: aaRS, aminoacyl-tRNA synthetase(s); AC, anticodon; ACRS, artificially created restriction site; ASL, anticodon stem and loop; bp, base pair; MELAS, mitochondrial encephalomyophathy, lactic acidosis, and stroke-like episodes; $\mathrm{mt}$, mitochondrial; $\mathrm{nt}$, nucleotide; $\mathrm{rho}^{-}$, large $\mathrm{mt}$ DNA deletion; $\mathrm{rho}^{+}$, complete mt DNA; rho ${ }^{\circ}$, absent mt DNA; WC, Watson-Crick; WT, wild-type.

${ }^{4}$ Corresponding author.

E-mail: silvia.francisci@uniroma1.it.

Article published online ahead of print. Article and publication date are at http://www.rnajournal.org/cgi/doi/10.1261/rna.2878711. demonstrated that targeted tRNA mutations can indeed be achieved using this technique.

Many $\mathrm{mt}$ diseases are due to base substitutions in $\mathrm{mt}$ tRNA genes, resulting in the impairment of $\mathrm{mt}$ protein synthesis and consequent OXPHOS defects. Pathogenic mutations have been identified in every human mt tRNA but three genes contain almost $50 \%$ of the known pathogenic substitutions: tRNA ${ }^{\text {Ile }}$, tRNA ${ }^{\text {Leu }}$ (UUR), and tRNA ${ }^{\text {Lys }}$ (www.mitomap.org). These mutations are distributed along the entire tRNA structure but anticodon nucleotides are least affected, probably because substitution at these positions would lead to cellular consequences too severe to permit cell growth (Wittenhagen and Kelley 2003).

The molecular and cellular consequences of several base substitutions in mt tRNA genes have been widely studied in yeast (Barrientos 2003) by both exploiting randomly occurring mutations (Zennaro et al. 1989; Francisci et al. 1998; Rohou et al. 2001) and generating biolistic mutants bearing substitutions equivalent to those causing human neurodegenerative diseases with different degrees of gravity (Feuermann et al. 2003; Montanari et al. 2008; De Luca 
et al. 2009). Defective yeast mutant phenotypes, defined by comparison with WT cells, include the following: absent, delayed or temperature sensitive growth on respiratory carbon sources, which requires functional mitochondria; high production of rho ${ }^{\circ}$ cells; and presence of punctuated mitochondria. Moreover, the effect of mutations on the tertiary structure and aminoacylation state of tRNA molecules can be readily recognized based on the difference in electrophoretic mobility between correctly folded and misfolded tRNAs, and between uncharged and aminoacylated tRNAs, respectively (Francisci et al. 2005). The absence or reduced level of mutated tRNA molecules has been ascribed to their instability (De Luca et al. 2009).

We have previously shown that the severity of phenotypes of human equivalent yeast mutants varies in different nuclear genetic contexts and reflects the gravity of the diseases observed in human (Feuermann et al. 2003; De Luca et al. 2006; Montanari et al. 2008). As an example, yeast cells bearing substitutions equivalent to those responsible for very severe pathologies (i.e., MELAS) do not respire in any of the tested nuclear contexts (De Luca et al. 2006). Importantly, by transforming the mutants with multicopy plasmids overexpressing tRNA interactors (e.g., $\mathrm{mt}$ protein elongation factor EF-Tu or cognate aaRS) we were able to suppress the defective phenotypes determined by the mutations and partially restore the steady-state levels of mutated tRNAs (Feuermann et al. 2003; De Luca et al. 2006, 2009). More recently, analogous results have been obtained in patient cell lines and cybrids (Park et al. 2008; Rorbach et al. 2008; Sasarman et al. 2008; Li and Guan 2010). Additionally, we have shown that ValRS and LeuRS have cross-suppressive activities on $\mathrm{mt}$ tRNA $^{\text {Leu }}$ and tRNA ${ }^{\text {Val }}$ yeast mutants and that suppression of yeast defects can be obtained by overexpression of orthologous human factors (Montanari et al. 2010). Further, we have shown that a point mutation in mt LeuRS reducing strongly its catalytic activity does not impair the protein ability to act as a suppressor (De Luca et al. 2009). These results suggest that the suppressive effect of aaRS is mediated, at least partially, by their ability to interact with mutated tRNAs and stabilize their structure in a chaperone-like manner, allowing the tRNAs to be correctly recognized and aminoacylated by the endogenous cognate aaRS.

In the present work we extend our investigations aimed at understanding the molecular basis and cellular phenotypes of $\mathrm{mt}$ diseases by introducing into the $\mathrm{AC}$ loop of yeast $\mathrm{mt}$ tRNA ${ }^{\text {Ile }}$ the T32C and T33C base substitutions (standard numbering, Sprinzl and Vassilenko 2005). These are equivalent to human mutations m.4290T $>C$ and m.4291T $>$ C, associated with progressive encephalopathy (Limongelli et al. 2004) and with a metabolic syndrome and hypomagnesaemia (Wilson et al. 2004), respectively. We use the term equivalent to indicate a mutation occurring at the same position and involving the same base substitution. The mt tRNA ${ }^{\text {Ile }}$ gene hosts a high number (14) of the mutations that have been associated with human diseases (www.mitomap.org) and is significantly similar in human and S. cerevisiae (see Fig. 1). Mutations at positions 32 and 33 are of special interest because the ability of suppressor molecules to rescue defective phenotypes associated with mutations occurring in the AC loop has not been investigated before.

We characterized in detail the yeast phenotypes determined by the introduction of the $\mathrm{T}>\mathrm{C}$ mutation at position 32 or 33 of $\mathrm{mt} \mathrm{tRNA}^{\mathrm{Ile}}$. We show that the phenotypes caused by the $\mathrm{T} 32 \mathrm{C}$ mutation are far more serious than those resulting from mutation $\mathrm{T} 33 \mathrm{C}$, mirroring the relative severity of the diseases associated with the equivalent human mutations m.4290T $>C$ and m.4291T $>C$, respectively. Overexpression of nuclear genes encoding tRNA interactors previously shown to act as suppressors toward yeast mt tRNA mutants revealed that aaRS, but not EF-Tu, were able to suppress the defective phenotype generated by the T32C and T33C mutations. We performed computational analyses of tRNA gene sequences and of known threedimensional crystallographic structures of tRNA molecules to understand the molecular bases of the observed phenotypes, and propose explanations in terms of structural changes occurring in tRNA ${ }^{\text {Ile }}$ mutants and/or impairment of their functional interactions with aaRS, ribosome, or other enzymes.

The results of this work allowed us to clarify some interesting features of mutations located in the AC loop of tRNA molecules, which had not been investigated in vivo before, and to extend to mutations $\mathrm{T} 32 \mathrm{C}$ and $\mathrm{T} 33 \mathrm{C}$ in $\mathrm{mt}$ tRNA $^{\text {Ile }}$ the relationship between the severity of human and yeast phenotypes associated with equivalent mutations in mt tRNA genes, which had been previously established for a number of mutations occurring in different tRNAs. In addition, we were able to enlarge the collection of tRNA positions that can be suppressed by overexpression of noncognate, as well as cognate, aaRS and may, therefore, be targeted by novel therapeutic strategies based on this feature.

\section{RESULTS}

\section{Base substitutions 32 and 33 in mt tRNA ${ }^{\text {Ile }}$ determine widely different phenotypes}

Examination of the cloverleaf structures of S. cerevisiae and human mt tRNA ${ }^{\text {Ile }}$ highlights the existence of significant overall similarities between the two molecules (Fig. 1). Both tRNAs belong to class I (i.e., they have short variable loops), and have AA stems, T stems, T loops, AC stems, and $A C$ loops of the same length. The sequence identity in the structurally conserved regions (comprising the whole molecules except the D and V loops) is $56 \%$ and the bp interactions within the stems are highly conserved both in number and type (i.e., WC vs. non-WC bps). The stability 

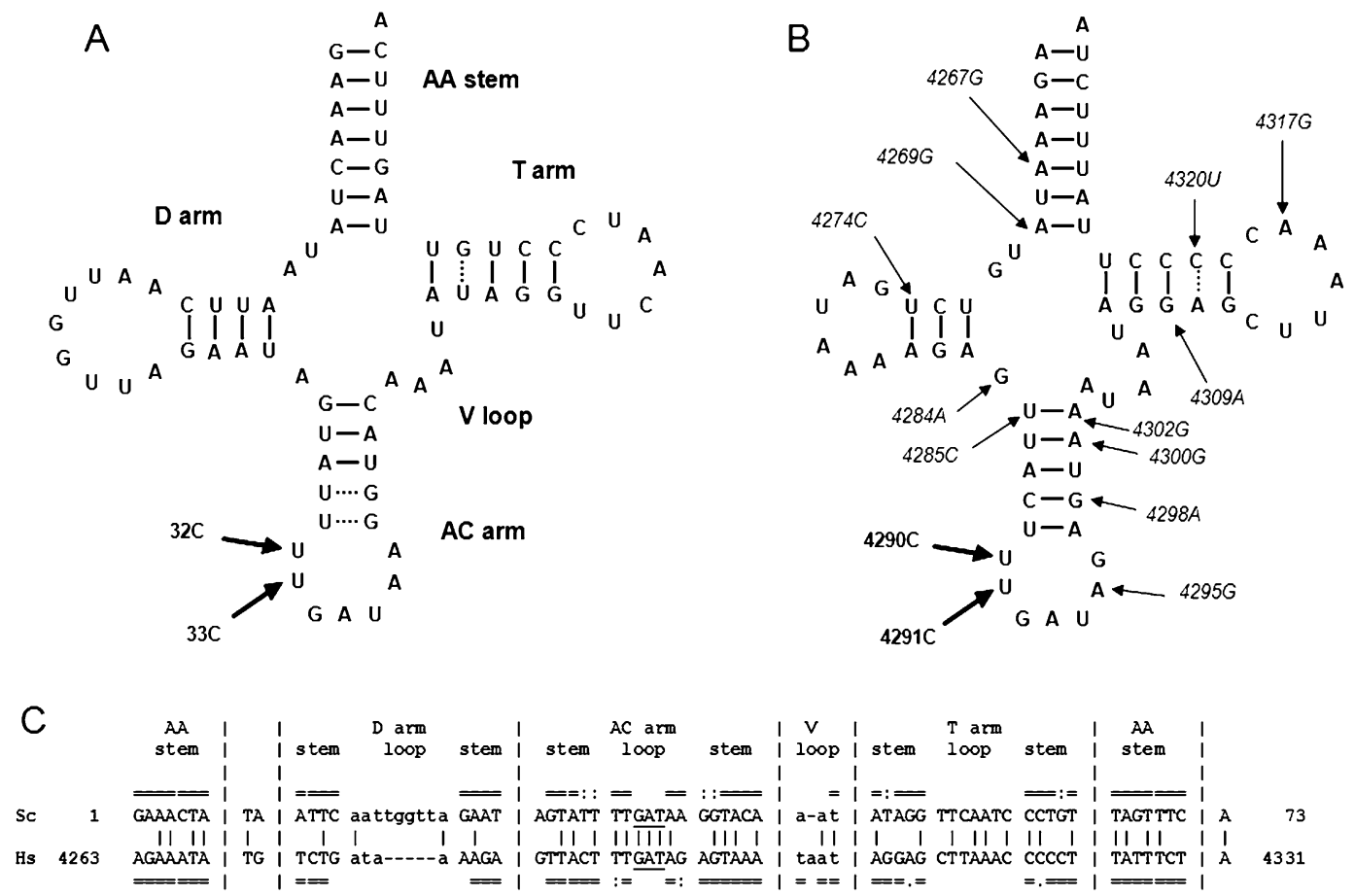

FIGURE 1. Comparison between yeast and human $m$ t tRNA ${ }^{\text {Ile }}$. Cloverleaf structures of yeast $(A)$ and human $(B)$ tRNA ${ }^{\text {Ile }}$. WC $(G-C$ and $A-U)$ and non-WC (G-U and A-C) bps are indicated by continuous and dotted lines, respectively. Mutations introduced in yeast mt DNA by biolistic procedures $(A)$ and human mutations $4290 \mathrm{C}$ and 4291C $(B)$, discussed in this work, are highlighted by thick arrows. Additional mt tRNA ${ }^{\text {Ile }}$ mutations correlated with pathologies are shown in italic and indicated by thin arrows. $(C)$ The yeast (Sc) and human (Hs) tRNA ${ }^{\text {Ile }}$ gene sequences are aligned as in the "Compilation of tRNA sequences and sequences of tRNA genes" database (Sprinzl and Vassilenko 2005). Nucleotides involved in WC (G-C and A-U) and non-WC (G-U and A-C) bps are indicated by the symbols "=" and ":", respectively. Identical nucleotides between the two sequences are indicated by vertical bars. Nucleotides that do and do not belong to the structurally corresponding regions between the two molecules are upper- and lowercase, respectively.

of the AC stems of yeast and human tRNA ${ }^{\text {Ile }}$ is not expected to be significantly different, since the only difference between them lies in the presence of two wobble G-U bp in the yeast molecule and two WC A-T bp in the human one, and the energy of $\mathrm{G}-\mathrm{U}$ and $\mathrm{A}-\mathrm{T}$ bp are quite comparable (Šponer et al. 1996). The AC loop differs in the identity of 1 out of $6 \mathrm{nt}$, namely A (in yeast) or G (in human) at position 38 , which is involved in a conserved, noncanonical bp interaction with the nucleotide at position 32 (Auffinger and Westhof 1999). However, in known tRNA structures the AC loop conformation is conserved and the interaction between nucleotides at positions 32 and 38 is present, even if the identity of the nucleotide occurring at these positions is variable (see the Results section, Structure and Sequence Analysis).

We introduced the mutations $\mathrm{T} 32 \mathrm{C}$ and $\mathrm{T} 33 \mathrm{C}$, adjacent to the $5^{\prime}$ of the AC in yeast mt tRNA ${ }^{\text {Ile }}$ (Fig. 1), using the biolistic procedure described in Feuermann et al. (2003) and Bonnefoy and Fox (2007). Figure 2 shows the growth phenotype of serial dilutions of WT and mutant cells observed after five days on $3 \%$ glycerol plates, incubated at $28^{\circ} \mathrm{C}$ and compared with the isogenic WT (MCC123).

In previous work (De Luca et al. 2006, 2009) we showed that the defective phenotype of all tested tRNA mutations varied depending on the nuclear context. Conversely, here we found that neither for the tRNA ${ }^{\text {Ile }} \mathrm{T} 32 \mathrm{C}$ nor for the T33C substitution the cytoduction crosses between the mutants with MCC123 nuclear context (kar1-1) and different rho $^{\circ}$ WT strains (D273-10B/A1, FF1210-6C, and W303-1B) changed the yeast growth phenotype (through-

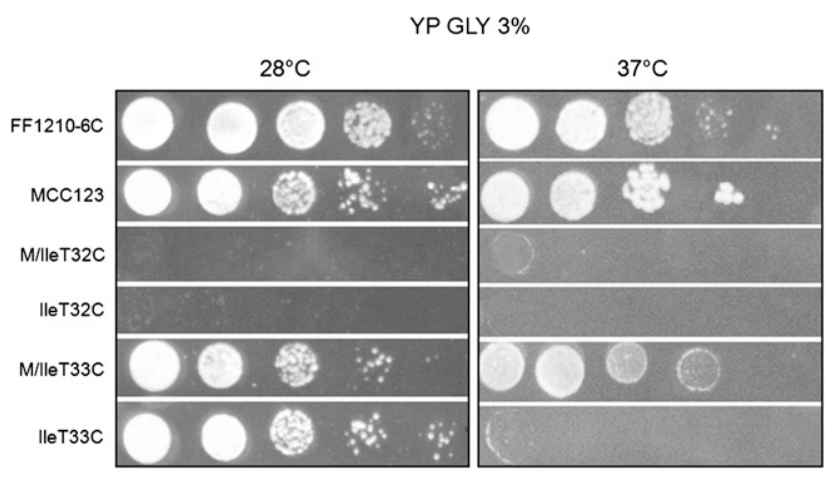

FIGURE 2. Growth phenotype of mt tRNA ${ }^{\text {Ile }} \mathrm{WT}$ and mutants. Serial dilutions of two WT (FF1210-6C and MCC123) and four (M/IleT32C, IleT32C, M/IleT33C, and IleT33C) mutant cells were spotted on glycerol $3 \%$ (GLY 3\%) YP medium plates and incubated at $28^{\circ} \mathrm{C}$ or $37^{\circ} \mathrm{C}$ for $3 \mathrm{~d}$. 
out the text, the names of mutants whose nuclear context is known are preceded by an uppercase letter followed by a slash, whereas the names of the spore mutants are not). In particular, the T32C base substitution conferred a very severe phenotype in all contexts, whereas the base substitution T33C did not affect glycerol growth. However, during the isolation of the latter mutant we observed that the diploid strain resulting from the cross between the rho ${ }^{-}$synthetic-MCC123 and FF1210-6C strains exhibited an improved growth compared with the WT (FF12106C) whereas the derivative spores had variable growth phenotypes. We dissected nine tetrads and analyzed eight spores, having the suitable auxotrophies, for their capability to grow in glycerol. The growth of five of these spores resulted to be thermosensitive and spore 10A (named IleT33C in the text) was chosen for further experiments. Sequencing of 1000 bps of the mt DNA region bearing the T33C mutation and 3500 bps of the DNA region containing the suppressor gene ISM1, coding for the cognate IleRS (see below), allowed us to verify that no additional mutations were present in the IleT33C mutant.

The respiration curves of the tRNA ${ }^{\text {Ile }}$ mutants grown in $0.25 \%$ glucose containing media at $28^{\circ} \mathrm{C}$, compared with two WT strains, are shown in Figure 3. In particular, Figure 3 shows the curves obtained for both the isogenic MCC123 mutants (M/IleT32C and M/IleT33C) and the spores (IleT32C and IleT33C) obtained from the sporulation of the diploid, rho $^{-}$synthetic MCC123 $\times$FF1210-6C. Cells bearing the T32C mutation did not respire in any of the tested nuclear contexts. On the contrary, the base substitution $\mathrm{T} 33 \mathrm{C}$ determined a limited decrease in $\mathrm{O}_{2}$ consumption compared with the WT.

The rho $^{-} / \mathrm{rho}^{\circ}$ production of the WT and tRNA ${ }^{\text {Ile }}$ mutants is shown in Table 1. It is interesting to note that the IleT32C spore originates the percentage of petites closest to the WT, despite the fact that it is not able to grow on glycerol media and does not respire (see Fig. 3 ). Conversely, both M/IleT33C and IleT33C spore strains, which do not show defective growth in glycerol at $28^{\circ} \mathrm{C}$ and have $\mathrm{O}_{2}$ consumption similar to the WT, show a higher petite percentage. DAPI staining was also performed to

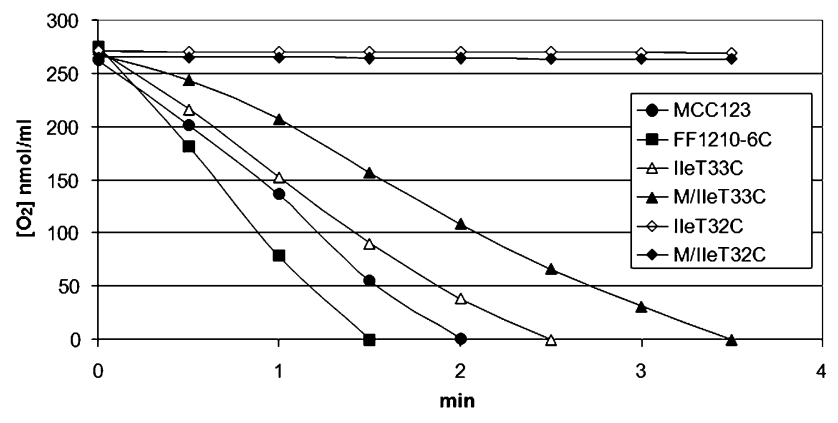

FIGURE 3. $\mathrm{O}_{2}$ consumption of two WT (MCC123 and FF1210-6C) and IleT33C, M/IleT33C, IleT32C, and M/IleT32C mutant strains.
TABLE 1. Petite percentage of strains after an overnight growth in $2 \%$ glucose containing YP media and oxygen consumption rate values per minute for $0.03 \mathrm{~g}$ of cells

\begin{tabular}{lcc}
\hline Strains & $\mathrm{Rho}^{-}, \mathrm{Rho}^{\circ}$ percentage & $\mathrm{O}_{2} \mathrm{nmol} / \mathrm{min}$ \\
\hline MCC123 & 2 & $137.2 \pm 8.6$ \\
FF1210-6C & 2.5 & $168.3 \pm 10.5$ \\
IleT32C & 6 & - \\
M/lleT32C & 20 & - \\
IleT33C & 12 & $109 \pm 12$ \\
M/lleT33C & 15 & $80.7 \pm 3.8$ \\
\hline
\end{tabular}

confirm the presence of mt DNA in $\mathrm{rho}^{-}$and its absence in rho ${ }^{\circ}$ cells.

\section{Effect of suppressor molecules on growth}

We have previously shown that suppression of defective phenotypes associated with single nucleotide substitutions in yeast tRNA ${ }^{\text {Val }}$ and tRNA ${ }^{\text {Leu }}$ (UUR) mutants can be obtained by overexpression of the $\mathrm{mt}$ protein elongation factor EF-Tu, or ValRS or LeuRS, as well as of orthologous human enzymes (Montanari et al. 2010). Both ValRS and LeuRS were able to suppress defects due to mutations in the cognate tRNAs and also showed cross-suppressing activities on tRNA ${ }^{\mathrm{Leu}}(\mathrm{UUR})$ and tRNA ${ }^{\mathrm{Val}}$ mutants harboring the $\mathrm{T}>\mathrm{C}$ substitution at position 25 .

To investigate whether the cross-suppressing activity of these proteins could be extended to other mutants, we transformed both tRNA ${ }^{\text {Ile }}$ mutants investigated in this work with plasmids bearing the genes ISM1, NAM2, VAS1, and TUF1, coding for mt IleRS, LeuRS, ValRS, and EF-Tu, respectively. Figure 4 shows the growth of serial dilutions of WT, mutant and transformed M/IleT32C cells on glycerol-containing plates incubated for $3 \mathrm{~d}$ at $28^{\circ} \mathrm{C}$. The growth defect of the tRNA ${ }^{\text {Ile }}$ mutant was rescued not only by the cognate IleRS but also when yeast cells were transformed with multicopy plasmids bearing the NAM2 or VAS1 genes, although the cognate IleRS had the highest rescuing efficacy. All protein products were endowed with suppressor activity only when expression of the plasmid gene was induced by $0.1 \%$ galactose.

Suppression experiments were also performed with the IleT33C spore. The latter mutant does not have marker selection for the multicopy vector and the transformants were directly plated on glycerol-containing media (data not shown). The thermosensitive glycerol growth defect of this spore was suppressed without the addition of galactose, indicating that the milder defect determined by the T33C mutation can be rescued by lower levels of suppressors than those required by M/IleT32C.

The overexpression of TUF1 did not increase the ability of either tRNA ${ }^{\text {Ile }}$ mutant to grow on glycerol media (see Fig. 4). 


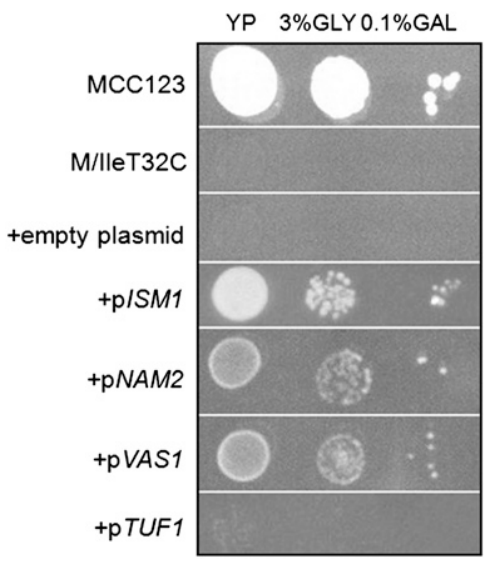

FIGURE 4. Suppression of mutant growth defective phenotype. Serial dilutions of WT (MCC123), M/IleT32C mutant, and the same mutant transformed with multicopy empty plasmid and multicopy plasmid bearing the genes ISM1, NAM2, VAS1, and TUF1 (coding for mt IleRS, LeuRS, ValRS, and EF-Tu, respectively) were spotted on 3\% glycerol plate with the addition of $0.1 \%$ galactose and incubated at $28^{\circ} \mathrm{C}$ for $3 \mathrm{~d}$. At $37^{\circ} \mathrm{C}$ aaRS showed the same suppression effects.

\section{Molecular effects of mutations and of suppressor genes (Northern analyses)}

In Figure 5 we report the Northern analysis of RNAs extracted from the purified mitochondria of tRNA ${ }^{\text {Ile }}$ mutant cells grown on YP medium containing $0.25 \%$ glucose, and from the same mutants transformed with suppressor genes. The RNAs were loaded in 10\% acrylamide partially denaturing sequencing gels, and the electrophoresis was conducted in acidic conditions.

In both the M/IleT32C mutant and IleT32C spore, not only was the mutated tRNA undetectable but the mutation produced a generalized absence of $\mathrm{mt} t \mathrm{tRNAs}$ (i.e., not only Ile and Gly, shown in Fig 5A, but also Leu and Asp, data not shown). Only when one of the suppressor genes (i.e., pISM1, pNAM2, or $\mathrm{p} V A S 1)$ was overexpressed, could mutated tRNAs be detected (Fig. 5A). However, the steady-state level of tRNA ${ }^{\text {Ile }}$ in transformant cells was not completely restored, as shown by the fact that to visualize the tRNA we had to load $40 \mu \mathrm{g}$ of total RNAs extracted from the mutant strains versus $10 \mu \mathrm{g}$ from the WT. The relative amounts of tRNAs present in cells containing different suppressor aaRS were quantified by Phoretix Analysis, and the level of tRNA ${ }^{\text {Ile }}$ was found to be threefold lower in cells rescued by the noncognate synthetases ( $\mathrm{pNAM2}, \mathrm{p} V A S 1)$. This result is in agreement with growth suppression experiments (Fig. 4) and indicates that the amount of tRNA ${ }^{\text {Ile }}$ in M/IleT32C cells rescued by PISM1, in spite of being fourfold lower than that extracted from WT cells, is sufficient to support glycerol growth and, therefore, $\mathrm{mt}$ protein synthesis. We investigated whether the inability to detect mt tRNAs in IleT32C cells by Northern analysis was limited to the examined $\mathrm{mt}$ tRNAs or extended to other RNAs coded by the mt genome, and found that the mt transcription product OXI2 (coding for subunit 3 of cytochrome oxidase) was also undetectable (data not shown).

For the IleT33C spore, which had shown thermosensitive growth, we extracted total mt RNAs either from cells grown at $28^{\circ} \mathrm{C}$ or from an aliquot of the same culture shifted for 4 $h$ at the nonpermissive temperature of $37^{\circ} \mathrm{C}$ (Fig. 5B, upper panel). Compared with the WT, at the permissive temperature tRNA ${ }^{\text {Ile }}$ was produced in normal amounts whereas it was undetectable in cells shifted at $37^{\circ} \mathrm{C}$. Hybridization with the GLY-probe showed that the amount of tRNA ${ }^{\text {Gly }}$ was similar to the WT, suggesting that the mechanism underlying the defect (e.g., instability; degradation; steadystate level reduction; etc.) occurring in the mutated tRNA $A^{\text {Ile }}$ at the nonpermissive temperature was specific for tRNA ${ }^{\text {Ile }}$ itself. The amount of tRNA ${ }^{\text {Ile }}$ extracted from M/IleT33C cells grown at $28^{\circ} \mathrm{C}$ and $37^{\circ} \mathrm{C}$ were similar to those obtained from WT cells.

Compared with the WT molecule, both T32C and T33C tRNA ${ }^{\text {Ile }}$ exhibited faster electrophoretic migration, indicating that a conformational change has occurred that is not compensated for in suppressed cells (Fig. 5A,B, upper panels). The acylation state of mutated tRNAs was also investigated by comparing their migration pattern to that of WT molecules. The T33C mutation does not impair aminoacylation of tRNA ${ }^{\text {Ile }}$, as inferred by the change in electrophoretic mobility observed following the deacylation treatment (Fig. 5B, lanes D). Aminoacylated tRNA ${ }^{\text {Ile }}$ was also present in M/IleT32C cells when suppressor genes were overexpressed.

\section{Structure and sequence analysis}

To investigate the effect of mutations on the tRNA ${ }^{\text {Ile }}$ molecule we performed an analysis of available tRNA three-dimensional structures and gene sequences (for details, see Supplemental Tables S1-S5).

Frequencies of nucleotides at positions 32 and 38 in $t R N A$ gene sequences

In tRNA gene sequences, the nucleotide at position 32 is almost always a pyrimidine, with a preference for $\mathrm{C}$ (present in both yeast and human mt tRNAIle mutants containing the T32C substitution) in almost all species and cell compartments. The most frequent nucleotide at position 38, which interacts with nucleotide 32 in tRNA structures (Auffinger and Westhof 1999), is A (present in yeast $\mathrm{mt}$ tRNA $^{\text {Ile }}$ ) followed by pyrimidines, while G (as in human $\mathrm{mt}$ tRNA $^{\text {Ile }}$ ) is very rare or completely absent (Supplemental Table S4). The most commonly occurring nucleotide pair at positions $32-38$ is $\mathrm{C}-\mathrm{A}$ followed by $\mathrm{T}-\mathrm{A}$, pyrimidinepyrimidine pairs and T32-G38. Remarkably, the C32-G38 pair (present in human $\mathrm{mt}$ tRNA ${ }^{\text {Ile }}$ containing the m.4290T $>$ C mutation) is almost completely absent (Supplemental Table S5). 

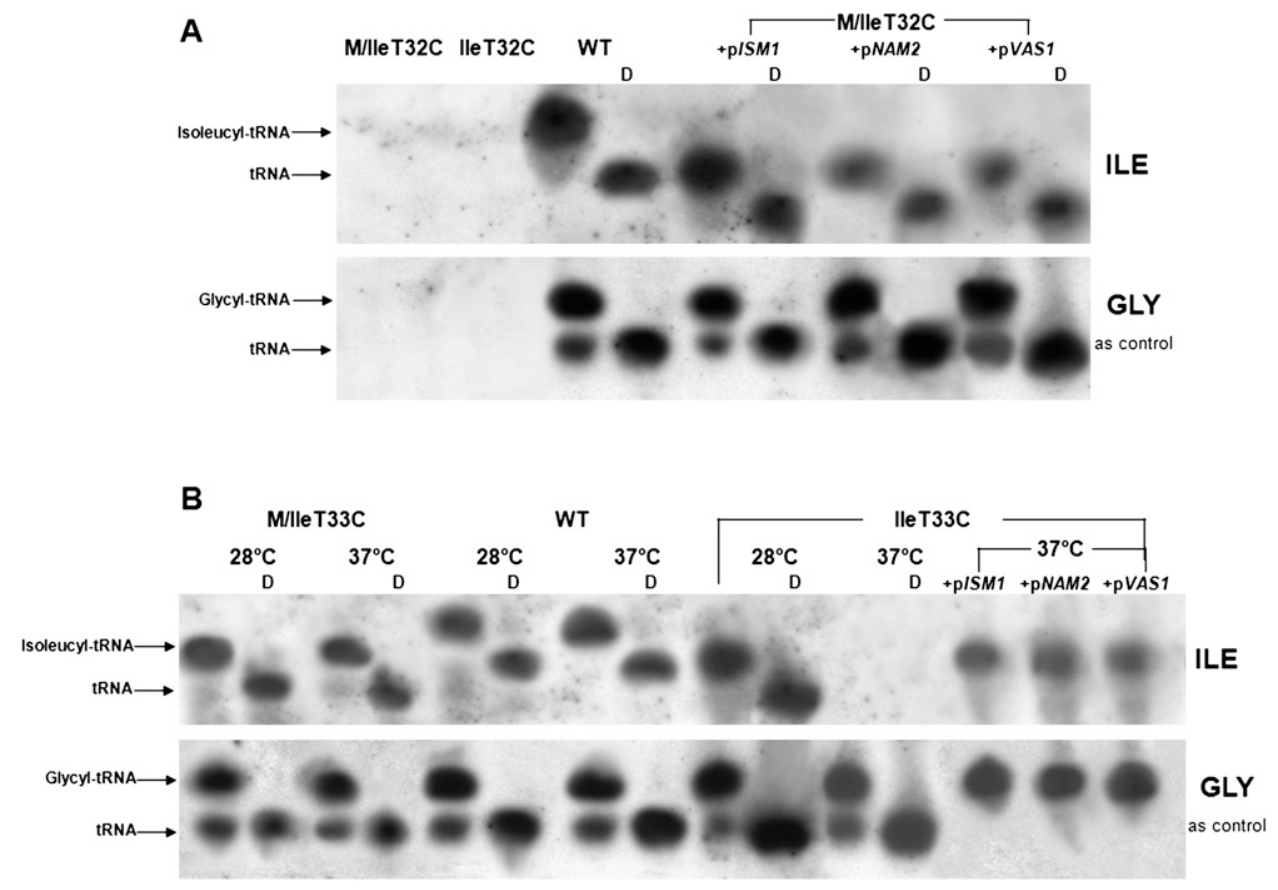

FIGURE 5. Northern blot analyses of mt tRNA ${ }^{\text {Ile }}$. (A) Total RNAs were extracted under acidic conditions from purified mitochondria of WT (MCC123), IleT32C, and the same mutant transformed with plasmids overexpressing the ISM1 and VAS1 genes. (B) The same experiment was performed with RNAs of IleT33C and of the same mutant transformed as in A. Cells were grown in YP medium containing $0.25 \%$ glucose at $28^{\circ} \mathrm{C}$. Half of the IleT33C thermosensitive mutant culture was also shifted for $4 \mathrm{~h}$ at $37^{\circ} \mathrm{C}$ before mitochondria extraction. WT $(10 \mu \mathrm{g})$ and mutant $(40 \mu \mathrm{g})$ native $\mathrm{mt}$ RNAs were fractionated on partially denaturing $10 \%$ polyacrylamide-urea acidic gels either directly or after alkaline treatment to deacylate the tRNAs (lanes $D$ ). Hybridization was with the $5^{\prime}$ end-labeled tRNAIle-probe (top panel) or with the $5^{\prime}$ end-labeled tRNAGly-probe (bottom panel), as control.

Interestingly, the mt tRNA ${ }^{\text {Ile }}$ data set shows peculiar preferences for nucleotides at positions 32 and 38 and for the 32-38 nt pair (Supplemental Tables S4, S5). The most frequently occurring nucleotides are T32 (present in both yeast and human WT mt tRNA ${ }^{\text {Ile }}$ ) and G38 (present in human $\mathrm{mt} \mathrm{tRNA}^{\text {Ile }}$ ). T-G is the most frequent nucleotide pair at positions $32-38$, followed by $\mathrm{T}-\mathrm{A}$, which is slightly more abundant than the "canonical" $\mathrm{C}-\mathrm{A}$. The mammalian $\mathrm{mt}$ tRNAIle data set has even more stringent preferences for T32 and G38, both of which are 100\% conserved.

\section{Interactions of nucleotides at positions 32 and 38 in $t R N A$} three-dimensional structures

In the available structures of free tRNA molecules, both nucleotides 32 and 33 establish several intramolecular interactions (see Supplemental Tables S2, S3). These are highly conserved in tRNA complexes with EF-Tu and ribosome, where the AC loop maintains its native structure, and only partially conserved in their complexes with the cognate aaRS, where the AC loop is distorted, several bases are moved out, and the majority of interactions of nucleotides 32 and 33 are replaced by different interactions within the tRNA molecules or with the enzyme.

In free tRNAs and in their complexes with EF-Tu or ribosome (Supplemental Table S2A) the nucleotide at position 32 is stacked between nucleotides 31 and 33, and nucleotides 32 and 38 form a noncanonical bp that is conserved even if the identity of the involved nucleotides is variable (Auffinger and Westhof 1999, and references therein). The base of nucleotide 38, in turn, is stacked between nucleotides 37 and 39 (Supplemental Table S2B). When the nucleotide pair at positions $32-38$ is $\mathrm{U}-\mathrm{A}$ (as in WT yeast mt tRNA ${ }^{\text {Ile }}$ ), a hydrogen bond is formed between the $\mathrm{O}_{2}$ atom of $\mathrm{U} 32$ and the $\mathrm{N6H}$ atom of A38 (Fig. 6A). When the nucleotide pair at positions $32-38$ is $\mathrm{C}-\mathrm{A}$ (as in the yeast mutant T32C) the $\mathrm{O}_{2}$ atom of C32 establishes an analogous interaction with $\mathrm{A} 38$, and an additional hydrogen bond is formed between the N3 atom of $\mathrm{C} 32$ and the $\mathrm{N} 3 \mathrm{H}$ atom of A38 (Fig. 6C). This second interaction cannot take place in the presence of U32, whose N3 atom is linked to a hydrogen atom $(\mathrm{N} 3 \mathrm{H})$ and, therefore, is not available to act as a hydrogen bond acceptor. The difference in hydrogen bond interactions established by U32 and C32 suggests that the $\mathrm{T} 32 \mathrm{C}$ mutation might affect the relative arrangement of the 32 and $38 \mathrm{nt}$ and, through this, the conformation of the AC loop.

While the AC arm does not interact with EF-Tu in the tRNA complex structures, it plays a major role in ribosome binding, which is contributed by hydrogen bonds involving the sugar-phosphate backbone atoms of nucleotides 32 

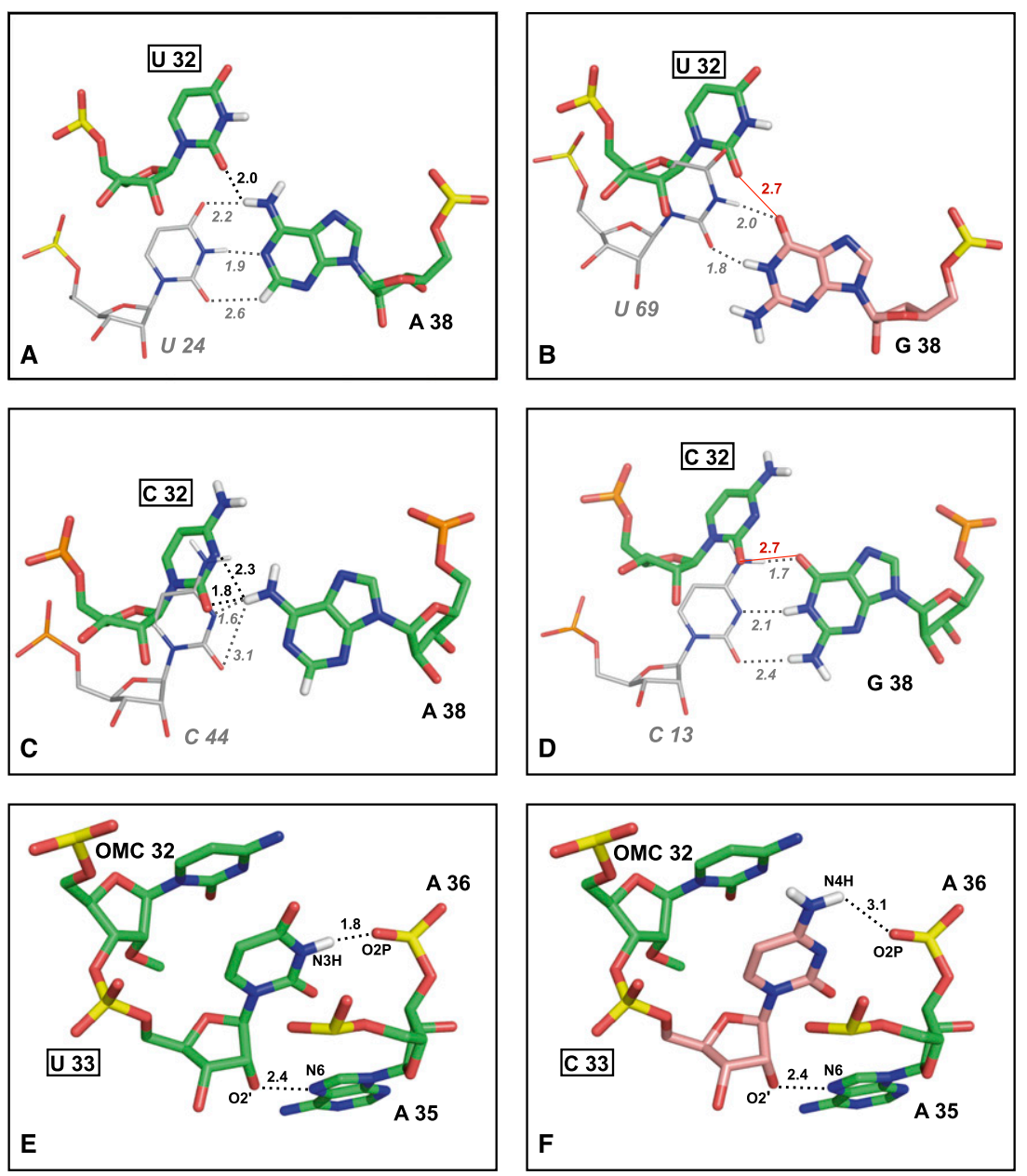

FIGURE 6. Structural role of U32 and U33. $(A, C)$ Noncanonical hydrogen bond interactions U32-A38 and C32-A38, respectively. $(B, D)$ Modeled U32-G38 and C32-G38 interactions, respectively. Nucleotides 32 and 38 are shown as sticks and colored by atom type: $\mathrm{O}$, red; $\mathrm{N}$, blue; H, white; P, yellow; C, green for the experimentally determined nucleotides U32, C32, and A38, salmon for the modeled G38. For comparative purposes, nucleotides U24, U69, C44, and $\mathrm{C} 13$, whose $\mathrm{C}$ atoms are colored gray $(A, B, C, D$, respectively), show the position that $\mathrm{U} 32$ or C32 would assume if they formed canonical WC bps with the nucleotide at position 38. (E) Three conserved intramolecular interactions of U33, namely (i) stacking of the uracil base between the base of nucleotide 32 and the phosphate moiety of nt 35; (ii) hydrogen bond of the ribose 2 '-OH with a nitrogen atom of the base at position 35; and (iii) hydrogen bond of the uracil N3H with $\mathrm{O} 2 \mathrm{P}$ of nucleotide 36. (F) Hypothetical interactions of the modeled C33: The first two interactions of $\mathrm{U} 33$ are maintained, but the hydrogen bond of $\mathrm{N} 3 \mathrm{H}$ is lost, and possibly replaced by a hydrogen bond involving $\mathrm{N} 4 \mathrm{H}$. Nucleotides 32, 33, 35 and the phosphate group of A36 are shown as sticks and colored by atom type as in previous panels $(\mathrm{C}$ atoms are green for the experimentally determined nucleotides U32, C32, U33, and A35, and salmon for the modeled C33). Hydrogen bonds are indicated by dashed lines and repulsive interactions by red lines. Distances $(\AA)$ are also shown. The structures used to produce this picture are reported in Materials and Methods. tal Table S2A,B). While the base atoms of both nucleotides 32 and 38 interact with class II aaRS, the nucleotide at position 32 does not interact with class I enzymes and the interactions of nucleotide 38 are mediated by sugarphosphate backbone atoms. This suggests that the effect of the T32C substitution on tRNA $^{\text {Ile }}$ aminoacylation, if any, is likely to be mediated by conformational changes of the AC loop rather than by the loss of base-specific interactions.

No tRNA structure where the nucleotide at position 38 is $\mathrm{G}$ (as in WT human $\mathrm{mt}$ tRNA ${ }^{\mathrm{Ile}}$ ) is available, therefore the A38G substitution was modeled into well determined tRNA structures containing U32 or C32, respectively. Following the A38G substitution, the hydrogen bond interaction established by the $\mathrm{O}_{2}$ atom of $\mathrm{U} 32$ or $\mathrm{C} 32$ with the $\mathrm{N} 6 \mathrm{H}$ atom of $\mathrm{A} 38$ (Fig. 6A,C) is replaced by a repulsive interaction with the $\mathrm{O} 6$ atom of G38 (Fig. 6B,D). This unfavorable interaction is likely to be relieved by the assumption of a 32-38 pair orientation different from those established by U32-A38 and C32-A38 pairs in known structures, and possibly involving the formation of canonical WC hydrogen bonds between $\mathrm{G} 38$ and $\mathrm{U} 32$ or C32 (see Fig. 6B,D).

Frequencies of nucleotides at position 33 in $t R N A$ gene sequences

Sequence analysis shows that $\mathrm{T}$ at position 33 is $100 \%$ conserved in tRNA ${ }^{\text {Ile }}$ from all species and compartments and largely predominant in all tRNA gene data sets (Supplemental Table S4). In the very few sequences where $\mathrm{T}$ is not present the nucleotide at position 33 is generally $\mathrm{C}$ or, very rarely, and never in yeast, human, or mammalian sequences, G or A. and/or 38. Consequently, putative conformational changes affecting the conformation of the AC loop may affect the interaction with the ribosome as well.

In some tRNA complexes with class I (like IleRS) or class II aaRS, the interactions of nucleotide 32 and 38 observed in free tRNAs and in EF-Tu or ribosome complexes are not conserved, and new base-base hydrogen bonds with other AC loop nucleotides are often established (see Supplemen-
Interactions of the nucleotide at positions 33 in $t R N A$ three-dimensional structures

In three-dimensional structures of tRNA molecules in the free state U33 is part of a conserved structural motif called "U-turn," consisting in a change of direction of the polynucleotide backbone and stabilized by three intramolecular non-WC interactions (Ashraf et al. 1999; Auffinger and 
Westhof 2001), which are conserved in tRNA complexes with elongation factor $\mathrm{Tu}(\mathrm{EF}-\mathrm{Tu})$ or ribosome (see Fig. 6E; Supplemental Table S3). Most of these interactions are mediated by the ribose-phosphate backbone or by atoms of the uracil base that are also present in cytosine (Supplemental Table S3) and might, therefore, be established by C33 as well. However, the conserved hydrogen bond between the $\mathrm{N} 3 \mathrm{H}$ atom of the uracil base, which is not present in cytosine, and the phosphate moiety of the nucleotide at position 36 would be lost following the U33C substitution (Fig. 6F). Indeed, modeling of the C33 (that is not present in known structures) showed that cytosine can be accommodated in the same conformation assumed by the WT uracil base, but the loss of the U33 (N3H)-nt $36(\mathrm{O} 2 \mathrm{P})$ hydrogen bond, and the possible formation of a new hydrogen bond involving the $\mathrm{N} 4 \mathrm{H}$ atom of $\mathrm{C} 33$ (Fig. 6F), may induce conformational rearrangements of the $\mathrm{AC}$ region affecting its functional performance.

The U33 (N3H)-nt $36(\mathrm{O} 2 \mathrm{P})$ hydrogen bond is conserved in tRNAs bound to the ribosome and might therefore be required to maintain the optimal AC conformation for an efficient translation process. Conversely, in aaRS complexes the U33-nt 36 hydrogen bond is generally lost, suggesting that the loss of the $\mathrm{N} 3 \mathrm{H}$ atom, consequent to the U33C mutation, would not affect the AC loop conformation required for aminoacylation to take place. Intermolecular interactions with ribosome or aaRS are unlikely to be significantly affected by the T33C mutation in other ways than those resulting from putative conformational changes. In fact, U33 contacts ribosome or aaRS almost exclusively through the sugar-phosphate backbone or the uracil $\mathrm{O}_{2}$ atom, which is also present in cytosine. Like U32, U33 does not interact with EF-Tu, which binds to the opposite side of the tRNA molecule.

\section{DISCUSSION}

Mt biogenesis and function depend on an intricate network of nucleo-mt interactions involving at least 1500 genes (Wallace and Fan 2009) that can modulate the activity of the respiratory chain. These numerous factors might explain the phenotypic variability and largely unpredictable course of mt diseases. Many, and possibly most, phenotypic differences among individuals may be elicited by alterations in gene expression and the underlying transcriptional regulation (Brem et al. 2002; Schadt et al. 2003; Yvert et al. 2003). These differences may take place at many levels, including transcription, messenger RNA processing, and stability, and the contribution of each level to respiratory efficiency is unknown.

Here we report the analysis of the yeast mutants bearing $\mathrm{mt} \mathrm{tRNA}^{\text {Ile }}$ substitutions at positions 32 and 33 in the AC loop, which are equivalent to human positions m.4290 and m.4291, respectively. Several mutations in human $\mathrm{mt}$ $\mathrm{tRNA}^{\text {Ile }}$ have been reported to be pathogenic and three of them, including the substitutions investigated in this paper, were found to be homoplasmic (www.mitomap.org). Heteroplasmy is the most frequent condition in patients and has been generally considered as the major cause of both the late onset of many $\mathrm{mt}$ illnesses and the tissue specificity of defects. However, several recent studies have revealed that homoplasmy, at least in somatic cells, might be a more frequent condition than what originally suggested (Lightowlers et al. 1997). This is particularly striking for tRNA mutations that have been reported to be homoplasmic in both affected and nonaffected members of the same family (McFarland et al. 2002; Limongelli et al. 2004). In this framework, an important feature of the yeast model is that it allows the effect of mutations in homoplasmic conditions to be studied.

In human, base substitution m.4290T $>C$ has been associated with severe encephalopathy, although it was found in all of the analyzed tissues. As an example, skeletal muscle morphology was normal, with a reduction of complexes I and IV. This substitution has been reported to have an extremely variable penetrance in the family where it was detected (Limongelli et al. 2004). The three children bearing the m.4290T $>C$ mutation had severe and fatal encephalopathy, while no data are available (e.g., about the levels of suppressor molecules) to explain the asymptomatic condition of the mother. Consistent with what observed in human, the tRNA ${ }^{\text {Ile }}$ T32C mutation, equivalent to m.4290T $>C$, conferred a very severe phenotype to yeast cells. It is interesting to note that the T32C substitution originated only between $6 \%$ and $20 \%$ petite colonies, depending on the nuclear context (Table 1). For the previously reported MELAS equivalent, not respiring yeast mutants, which lost mt DNA very rapidly and originated up to $80 \%$ petites (Feuermann et al. 2003; Montanari et al. 2008), the absence of mt tRNAs had been ascribed to the loss of mt DNA. Conversely, the absence of mt tRNAs determined by the T32C mutation, highlighted by the Northern experiment reported in Figure 5, cannot be entirely determined by the loss of mt DNA, given the low percentage of petite colonies, but may be contributed by the existence of feedback mechanisms connecting mt transcription and translation. Similar results have been obtained previously for the mutation A29G in tRNA ${ }^{\text {Leu }}$ UUR (De Luca et al. 2009).

The yeast mutation $\mathrm{T} 33 \mathrm{C}$ is equivalent to the human substitution m.4291T $>C$, which has been reported to be involved in a homoplasmic, age related, dyslipidemic, and hypomagnesemic metabolic syndrome (Wilson et al. 2004). In yeast, this substitution determines a moderately severe defect, a $12 \%-15 \%$ production of rho $^{\circ}$ cells and a thermosensitive glycerol growth phenotype, which could be observed only in definite combinations of genetic contexts. Indeed, we have previously observed that the same mutated mitochondria introduced into different rho $^{\circ}$ strains often produced different molecular phenotypes (De Luca et al. 
2006, 2009), which is reminiscent of the variable penetrance observed in patients harboring the same mt mutation. This phenomenon may be explained in terms of the different amounts of nuclear products related to tRNA functionality that are able to interfere with $\mathrm{mt}$ functions. Northern analysis (Fig. 5) showed that the spore T33C grown at $28^{\circ} \mathrm{C}$, while having similar amounts of aminoacylated tRNA $^{\text {Ile }}$ compared with the WT, did not contain detectable tRNA ${ }^{\text {Ile }}$ when grown for a few hours at $37^{\circ} \mathrm{C}$. This behavior was specific of tRNA ${ }^{\text {Ile }}$ and has been previously observed for other thermosensitive mutants (Zennaro et al. 1989; Francisci et al. 1998). The limited defects observed for the T33C mutation in defined genetic contexts parallel the peculiar distribution of the dyslipidemic syndrome in the 129 members of the maternal lineage kindred studied by Wilson et al. (2004). In other words, the penetrance of this syndrome might depend on the genetic context of the nucleus.

Suppression experiments were performed for mutant $\mathrm{M} / \mathrm{IleT} 32 \mathrm{C}$ and for the spore IleT33C, exhibiting a clearly defective growth phenotype. The cognate IleRS, as well as ValRS and LeuRS, all belonging to the same class (I) and subclass (a), were able to rescue the defective phenotypes associated with both mutations, the cognate IleRS showing the highest suppression activity. These results indicate that the cross-suppression activity of ValRS and LeuRS, previously shown for tRNA ${ }^{\text {Val }}$ and tRNA ${ }^{\text {Leu }}$ mutants, can be extended at least to mutations in tRNA ${ }^{\text {Ile }}$. This suggests that the cross-suppressive activity is likely to be a property of aaRS belonging to the same class and, possibly, subclass, and/or acting on tRNAs specific for similar aminoacids, such as leucine, isoleucine, and valine. Conserved motifs present in these different enzymes might be responsible for stabilizing the tRNA structure altered by mutations (Montanari et al. 2010). The suppressing effects of aaRS on the defects produced by mutations in tRNA molecules are shown in this paper to be regulated by the amount of suppressors available. This result has been obtained using multicopy vectors bearing the aaRS genes whose transcription is controlled by the GAL1 promoter (Fig. 4). The severe defective phenotype of M/IleT32C mutant was suppressed only in the presence of galactose. The requirement of variable amounts of suppressors to rescue respiratory defects has been previously observed for several tRNA ${ }^{\text {Leu }}$ UUR mutants with severely defective phenotypes (De Luca et al. 2006).

An important point arisen in this work is the lack of suppressing activity shown by the $\mathrm{mt}$ protein elongation factor EF-Tu. This factor had been previously found to be a general suppressor of the defects of our whole yeast tRNA mutant collection, comprising more than 20 strains bearing mutations dispersed in all tRNA regions, with the only exception of mutation G36A located in the AC loop of tRNA $^{\text {Arg2 }}$ (Francisci et al. 2005; S Francisci, A Montanari, C De Luca, and L Frontali, unpubl.). We have previously ascribed the rescuing effect of suppressor molecules to their ability to induce a native-like conformation in tRNA regions where mutations are located. Here we show that overexpression of TUF1 does not increase growth on glycerol media of either tRNA ${ }^{\text {Ile }}$ mutant. Since EF-Tu binding by tRNA molecules does not involve the AC loop, where the $\mathrm{T} 32 \mathrm{C}$ and $\mathrm{T} 33 \mathrm{C}$ mutations are located, but the region farthest apart from it in the tRNA structure, the lack of suppressive effect is probably not caused by the inability of EF-Tu to bind the mutated tRNA, but by the inability of this interaction to induce a native-like conformation in the AC loop. Conversely, the ability of aaRS to suppress defects caused by mutations occurring in the entire tRNA molecule, including the AC loop, may be ascribed to their ability to establish extensive interactions with essentially all tRNA regions.

Analysis of three-dimensional structures and gene sequences of tRNA molecules allowed us to propose explanations for the observed defects. Both yeast $\mathrm{mt}$ tRNA ${ }^{\text {Ile }}$ mutants were shown by Northern experiments to undergo conformational changes with respect to the WT molecules (Fig. 5). These conformational changes are likely to be determined by the replacement, in both mutants, of interactions occurring in the WT molecules with analogous, but not identical, interactions. In particular, in the tRNA ${ }^{\text {Ile }}$ containing the T32C substitution a new hydrogen bond is formed between C32 and A38 in addition to the T32-A38 hydrogen bond present in the WT molecule, and this might alter the geometry of the 32-38 pair (Fig. 6A,C). In the tRNA ${ }^{\text {Ile }}$ containing the T33C mutation, the strongly conserved hydrogen bond between a phosphate oxygen of the nucleotide at position 36 and U33 is lost and, possibly, replaced by a different hydrogen bond with C33. As mentioned above, conformational changes in the AC loop are unlikely to affect recognition by EF-Tu, but they may affect tRNA interactions with the ribosome, which establishes extensive contacts with this tRNA region. This hypothesis is supported by the results of previous biochemical studies, showing that the binding affinity of tRNA fragments toward the ribosome is significantly affected by the identity of nucleotides at positions 32 and 38 (Olejniczak et al. 2005 and references therein), and the affinity of a yeast tRNA $^{\text {Phe }}$ ASL construct containing the U33C substitution for the $30 \mathrm{~S}$ ribosome subunit is reduced to $5 \%$ of the unsubstituted ASL, a result that was ascribed to the loss of the conserved U33 hydrogen bond with the phosphate group of A36 described above (Ashraf et al. 1999). Alterations in tRNA affinity for the ribosome might affect the efficiency of protein synthesis and this might contribute, at least in part, to the observed yeast phenotypes (i.e., defects in $\mathrm{O}_{2}$ consumption and increased percentages of petite cells).

Northern experiments show that the tRNA ${ }^{\text {Ile }}$ bearing the T32C mutation is correctly aminoacylated in the presence of suppressors (Fig. 5A) but we cannot exclude that aminoacylation defects contribute to instability and/or 
degradation of the mutated molecule in their absence. Although the nucleotide at positions 32 is not directly in contact with class I aaRS residues in the analyzed structures of tRNA-aaRS complexes, the conformational variation occurred in the AC loop following T32C mutation might affect class I aaRS binding and aminoacylation. Additionally, the nucleotide at position 32 has been previously reported to be required for aminoacylation of Escherichia coli tRNA ${ }^{\mathrm{fMet}}$ by the cognate class I MetRS (Giegé et al. 1998), and class II aaRS residues interact with base atoms of nucleotide 32 in the analyzed structures. The tRNA ${ }^{\text {Ile }}$ bearing the T33C mutation is correctly aminoacylated (Fig. 6A), indicating that the conformational change occurred in the AC loop does not impair the ability of this mutant to interact with the cognate IleRS. Accordingly, structure analysis showed that both the intra- and intermolecular interactions established by U33 in the three-dimensional structures of aaRS complexes are likely to be maintained in the presence of $\mathrm{C} 33$.

In addition to the interactions observed in the available three-dimensional structures of tRNA complexes (e.g., with aaRS or ribosome), the nucleotides at positions 32 and 33 may establish interactions with different molecular partners, such as enzymes involved in tRNA maturation or posttranscriptional modification, and these interactions may be negatively affected by the T32C and/or T33C mutation as well. As an example, post-transcriptional modifications play an important role in both tRNA structure stabilization and function, in that they can be recognition elements for interacting partners such as aaRS or mRNA codons (Agris 1996), and decreased stability is the feature of mutated mt tRNA molecules that has been most commonly associated with pathogenesis (Levinger et al. 2004; Kirino and Suzuki 2005).

The structural considerations described above can be extended to the equivalent human mutations m.4290T $>C$ and m.4291T $>$ C. Indeed, modeling of G38 in known structures suggests that in its presence, as in the presence of $\mathrm{A} 38$, the $\mathrm{T} 32 \mathrm{C}$ mutation is likely to determine an alteration in the pattern of hydrogen bond interactions established by the pyrimidine at position 32 with the purine at position 38 (Fig. 6A-D). Additionally, recent work showed that the human mutation m.4290T $>\mathrm{C}$ (equivalent to position 32 ) and the murine mutation $\mathrm{m} .3739 \mathrm{G}>\mathrm{A}$ (equivalent to position 38) in mt tRNA ${ }^{\text {Ile }}$ have similar molecular effects (Moreno-Loshuertos et al. 2011). This supports the hypothesis that the main effect of the T32C mutation is the alteration of the base-base interactions with the nucleotide at position 38 present in the WT molecule and does not depend significantly on the specific base at position 38 .

Interestingly, analysis of tRNA gene sequences showed that the mt tRNA ${ }^{\text {Ile }}$ data set has peculiar preferences for the nucleotides occurring at positions 32 and 38 . The defective phenotypes generated by the substitution at position 32 of $\mathrm{T}$ (favored in $\mathrm{mt} t \mathrm{RNA} \mathrm{A}^{\text {Ile }}$ ) with $\mathrm{C}$ (which is most frequent in other tRNA data sets) suggest the existence of a relation- ship between the nucleotide present at this position and the amino acid specificity of the tRNA molecule. Indeed, previous in vitro studies have shown that the strength of ribosome binding of tRNA fragments differing only at positions 32 and 38 varied to a large extent, and this behavior was ascribed to the requirement to tune tRNA affinity for the ribosome to a uniform value (Olejniczak et al. 2005 and references therein).

In spite of the fact that the U33C mutation generates only a mildly severe phenotype and affects relatively few tRNA interactions, U33 is $100 \%$ conserved in tRNA ${ }^{\text {Ile }}$ and highly conserved in mt and cytoplasmic tRNA from all species (even if, when it is substituted, its most common replacement is $\mathrm{C}$ ). This underlines the complexity of the factors determining the pathogenic effects of a mutation and the limits of sequence conservation at sites affected by mutations as generally accepted criteria to infer pathogenicity (McFarland et al. 2004). Together with sequence analysis, the analysis of available three-dimensional structures can greatly contribute to overcome these limitations as well as increase our understanding of pathogenetic mechanisms.

The results presented in this work on the effect of substitutions in the AC loop of $m t \mathrm{tRNA}^{\text {Ile }}$, which had not been studied in vivo before, and the suppressing effect of cognate and noncognate aaRS clearly show the importance of combining experimental with structure and sequence analysis approaches to understand the molecular mechanisms at the origin of diseases and identify potential ways to interfere with them for therapeutic purposes.

\section{MATERIALS AND METHODS}

\section{Media, growth conditions, and respiration studies}

Strains were grown in YP complete medium (1\% yeast extract and $1 \%$ peptone from Difco) containing $3 \%$ glycerol or glucose $(2 \%$ or $0.25 \%)$. To induce Gal-promoter in the suppression experiments, $0.1 \%$ galactose was added to YP $3 \%$ glycerol plates. Minimal medium was $0.7 \%$ yeast nitrogen base (Difco), $5 \%$ ammonium sulphate, and $2 \%$ glucose, supplemented with the necessary auxotrophic requirements. For solid plates, 1.5\% agar (Difco) was added to the above media.

The production of $\mathrm{rho}^{-} / \mathrm{rho}^{\circ}$ colonies was monitored as follows: A large colony grown on a YP $2 \%$ glucose plate was inoculated in the same liquid medium. After overnight growth, the culture was diluted and plated on glucose, and large and small colonies were counted. To discriminate between $\mathrm{rho}^{-}$and $\mathrm{rho}^{\circ}$ colonies, DAPI staining was performed. In all of the examined cases, colonies with a small size, unable to grow on glycerol, were found to be devoid of mt DNA.

Respiration studies were performed using a Clark oxygen electrode (Hansatech Instruments). Large colonies, corresponding approximately to $0.03 \mathrm{~g}$ (wet weight) of cells, were collected from $2 \%$ glucose plates, grown for $4 \mathrm{~h}$ in fresh $0.25 \%$ glucosecontaining medium, and washed with $1 \mathrm{~mL}$ sterile water. After 
centrifugation the pellet was suspended in $1 \mathrm{~mL}$ of phosphate buffer $10 \mathrm{mM}, \mathrm{pH} 7.4$, containing $0.4 \%$ glucose, and loaded in the Reaction Vessel of the previously calibrated Oxygen Electrode Chamber. Endogenous oxygen consumption was measured for $5 \mathrm{~min}$. The rate values derived from at least three independent experiments and the standard deviation calculated for any point varied between 12 and $3.8 \mathrm{nmol} / \mathrm{mL}$.

\section{Strains, plasmids, mutagenesis, and biolistic transformation}

S. cerevisiae strains used in this study are as follows: D273-10B/A1 MAT $\alpha$, met $^{-}$ura3, $\Delta$ leu, $\mathrm{Kan}^{\mathrm{R}}$, $\mathrm{rho}^{\circ}$ and $\mathrm{rho}^{+}$(Berlani et al. 1980), FF1210-6C MAT $\alpha$, ura 1-2, rho $^{\circ}$ and rho $^{+}$(BolotinFukuhara et al. 1977), FF1210-6C MAT $\alpha$, ura 1-2, $\Delta$ leu, Can ${ }^{\mathrm{R}}$, $\mathrm{Kan}^{\mathrm{R}}$, $\mathrm{rho}^{\circ}$ and $\mathrm{rho}^{+}$(kindly provided by Prof. M. BolotinFukuhara), MCC123 MAT a, ade2, ura3-52, kar1-1, rho ${ }^{\circ}$ and rho $^{+}$ (Mulero and Fox 1993), MCC123 MAT a, ade2, ura3-52, $\Delta$ leu, kar1-1, $\mathrm{Kan}^{\mathrm{R}}$, $\mathrm{rho}^{\circ}$ and $\mathrm{rho}^{+}$(kindly provided by Prof. M. Bolotin-Fukuhara), YGM128 MAT $\alpha$, ura 1-2, $\Delta$ leu, $\mathrm{Can}^{\mathrm{R}}, \mathrm{Kan}^{\mathrm{R}}$, $\mathrm{rho}^{\circ}$ and $\mathrm{rho}^{+}$(Bolotin-Fukuhara et al. 1977), TF145 MAT $\alpha$, ade2-1, ura3-52, mit-OXI1-17 (Fox et al. 1991). The mutant strains were named with a first letter identifying the nuclear background followed by a slash, the three-letter code name of the amino acid indicating the tRNA gene, and the base substitution. The names of the spores analyzed in this work do not have the first letter because they are derived from the recombination of two different nuclear backgrounds (MCC123 and FF1210-6C) necessary to obtain biolistic mutants.

To obtain the mutants analyzed in this work, mt DNA from WT strain FF1210-6C was digested by the NsiI restriction enzyme and a fragment of 800 bps was cloned into the ptz18 vector in the PstI site.

Site-directed mutagenesis was performed using, for each substitution, two couples of mutagenic primers complementary to 18 to 54 residues of the tRNA ${ }^{\text {Ile }}$ gene (IleT32C+: $5^{\prime}$-GGTTAGAA TAGTATTCTGATAAGGTACAAATATAGG-3' and IleT32C-: 5' -CCTATATTTGTACCTTATCAGAATACTATTCTAACC-3', IleT33C+: 5' -GGTTAGAATAGTATTTCGATAAGGTACAAATATA GG-3' and IleT33C-: 5'-CTATATTTGTACCTTATCGAAATACT ATTCTAACC- $3^{\prime}$ ). Purified plasmid DNA from the mutagenesis was prepared and sequenced to confirm the presence of the point mutations.

The inserts containing the $\mathrm{T} 32 \mathrm{C}$ and $\mathrm{T} 33 \mathrm{C}$ mutations were subcloned into the pJM2 vector and utilized for biolistic transformation of the MCC123 $\mathrm{rho}^{\circ}$, as described in Feuermann et al. (2003). In order to recognize the recombinant mt mutant clone among the WT ones, (i) for the $\mathrm{T} 32 \mathrm{C}$ mutation we designed a specific ACRS primer (IleT32C/ACRSEcoRI+: $5^{\prime}$-TTCAATTGG TTAGAATAGAATT- $3^{\prime}$ ), which introduces certain mismatches in the amplified fragment producing a new EcoRI restriction cleavage site; (ii) for the T33C mutation we performed a TaqI digestion of the 460-bp PCR product amplified from five mixed colonies (utilizing ALA2+: 5'-GTTCAAGTCTCATTAACTCC-3' and $3^{\prime}$ ILE-: $5^{\prime}$-AACTATATATATTTATTATAAATTAAA-3 primers), in that the $\mathrm{T} 33 \mathrm{C}$ mutation introduces a new TaqI cleavage site in the tRNA ${ }^{\text {Ile }}$ gene. We obtained one fragment of 120 bps and two fragments of 170 bps from mutated PCR products, and only two fragments, of 120 and $340 \mathrm{bps}$, respectively, from WT PCR products.
The PCR reactions were performed directly on colonies in 50 $\mu \mathrm{L}$ and the thermocycler conditions were as follows: $5 \mathrm{~min}$ at $95^{\circ} \mathrm{C}$ for one cycle, followed by $30 \mathrm{sec}$ at $94^{\circ} \mathrm{C}, 1 \mathrm{~min}$ at $50^{\circ} \mathrm{C}$, and $1 \mathrm{~min}$ at $72^{\circ} \mathrm{C}$ for 30 cycles. The endonuclease digestion was performed with $5 \mu \mathrm{L}$ of each PCR product in a total volume of 20 $\mu \mathrm{L}$. The pattern of migration was observed on agarose-1000 gel (Invitrogen) 3\%, which allowed us to distinguish between the native PCR product and the digested one.

To confirm the presence of the single point mutations in each recombinant strain, the mt DNA region containing the tRNA ${ }^{\text {Ala }}$, tRNA $^{\text {Ile }}$, and RNA $^{\text {Tyr }}$ genes was amplified and sequenced. Additionally, for the IleT33C spore we sequenced 3500 bps of the DNA region bearing the ISM1 gene, coding for the IleRS.

Sequences were determined by Bio Molecular Research, Sequencing Service, Padova and compared with the complete $\mathrm{mt}$ DNA sequence reported by Foury et al. (1998).

Base substitutions T32C and T33C in tRNA ${ }^{\text {Ile }}$ were introduced in the MCC123 nuclear context and successively also in nuclear contexts W303-1B, FF1210-6C, and D273-10B/A1. These mutants were obtained by cytoduction crosses transferring mitochondria containing the mutated tRNA genes into different hho $^{\circ}$ nuclear backgrounds, according to the procedure described in De Luca et al. (2006).

Standard protocols (Sambrook et al. 1989) were used for E. coli and yeast transformations, as well as plasmid preparation.

The TUF1 gene was cloned with its own promoter in the multicopy plasmid YEpLAC181 (Rinaldi et al. 1997).

The NAM2 gene was cloned into the multicopy vector pFL44L (pENAM2) (Herbert et al. 1988).

The VAS1 gene was cloned into pCM262 (De Luca et al. 2009).

The ISM1 gene encoding for yeast mt Ile-RS was cloned into the multicopy vector pYES2.1/V5-His-TOPO (pYES2.1TOPO TA Expression Kit, Invitrogen) under the inducible Gall promoter. The fragment containing the ISM1 gene was amplified using the following primers: ISM+ $\left(5^{\prime}\right.$-ATGAAGCGCTCCAGGCTTGTG CCTC-3') and Sma1ISM- (5'-TCCCCCGGGGGATCAAGAC ATGAGATGATCCACAG-3') from genomic DNA.

\section{DNA and RNA manipulations}

All methods for nucleic acid preparations were as previously described by Francisci et al. (1998). For electrophoresis, blotting, and hybridization conditions, see Francisci et al. (2005) and references therein. Partially denaturing sequencing gels $(40 \mathrm{~cm}$ in length) can distinguish a single-base insertion or deletion in tRNAs (Sambrook et al. 1989).

Mt RNAs were extracted under acidic conditions (sodium acetate buffer $0.3 \mathrm{M}, \mathrm{pH}$ 5) from purified mitochondria of WT and mutant cells grown to mid-log phase in YP $0.25 \%$ glucose containing media at $28^{\circ} \mathrm{C}$, as described by Baldacci and Zennaro (1982). As far as the thermosensitive mutant is concerned, the culture was also shifted for $4 \mathrm{~h}$ at $37^{\circ} \mathrm{C}$ before mitochondria extraction.

Total native mt RNAs and alkaline-treated samples were separated in partially denaturing $10 \%$ polyacrylamide- $8 \mathrm{M}$ urea sequencing gels; the electrophoresis conditions and procedures are as described in Francisci et al. (2005) with minor modifications. To increase the separation between acylated and deacylated tRNAs, gel electrophoresis was run for $48 \mathrm{~h}$. We have previously shown that mutated tRNAs can have different electrophoretic 
mobility from WT tRNAs in partially denaturing conditions, and this behavior has been ascribed to structure alterations (Francisci et al. 2005). We loaded $10 \mu \mathrm{g}$ of mt RNA extracted from WT cells, while $40 \mu \mathrm{g}$ of total RNAs from mutants were loaded to visualize the mutated aminoacyl tRNA ${ }^{\text {Ile }}$ molecule.

\section{Hybridization condition and probes}

The RNA blotting was performed on Amersham's Hybond-XL membranes according to the protocol provided by the supplier.

The tRNAs were identified by hybridization with very specific probes (oligonucleotides ILE-: 5'-CCTATATTTGTACCTT ATC-3', and GLY2-: 5' -CGAACTCGCATTCAATGTTTGGAAG ACATCCAGTTTACC-3') 5' -end-labeled as described by Sambrook et al. (1989).

The absence of cytoplasmic contamination of the RNAs extracted from highly purified mitochondria was verified by hybridizing the blots with a $5^{\prime}$-end-labeled probe ( $5^{\prime}$-GCATCGTC AATGACTAGTCG-3') specific for the snR39 gene (Montanari et al. 2008) (data not shown).

\section{Structure and sequence analysis}

The three-dimensional structures of tRNA molecules analyzed in this work were downloaded from the PDB (Berman et al. 2000). A complete list of structures, including structure resolution and average B-factor in the 32-38 region (the lower these values, the higher the structure reliability in the AC loop region), is reported in Supplemental Table S1. The analyzed structures comprise (i) all available tRNA molecules in the free state; (ii) all complexes of tRNAs whose structure, from the same or a different species, is also available in the free state; (iii) all available structures of tRNA ${ }^{\text {Ile }}$. We excluded from our analysis redundant structures (i.e., structures of the same tRNA molecules in the same state of interaction as those in Supplemental Table S1 but determined at lower resolution) and the structure of yeast $\mathrm{tRNA}^{\mathrm{fMet}}$ in the free state (PDB ID: 1YFG) because the AC loop has high temperature factors (the average value in the $32-38$ region is 114) and the AC region is involved in lattice contacts that might induce the assumption of a nonphysiological conformation (Basavappa and Sigler 1991). A complete list of the interactions established by nucleotides at positions 32 and 33 in the analyzed structures is reported in Supplemental Tables S2 and S3. Structure visualization and analyses were performed using the software InsightII (Accelrys Inc.) and PyMOL (Schrödinger LLC). InsightII was also used to model the A38G and U33C mutations in well-determined tRNA structures by computationally replacing the adenine and uracil bases with guanine and cytosine, respectively. The only available tRNA structure in the PDB with C33, namely Methanococcus jannaschii tRNA $^{\text {Leu }}$ in complex with the modification enzyme tRNA- $\left(\mathrm{N}^{1} \mathrm{G} 37\right)$ methyltransferase (Trm5, PDB ID: 2ZZM), was considered not to be representative of the interactions of C33 in free tRNAs or in tRNA complexes with EF$\mathrm{Tu}$ or ribosome because the AC loop conformation is very different from the canonical one and the cytosine base is moved out toward the solvent and unable to establish intramolecular interactions.

The three-dimensional structures used to visualize the interactions of nucleotides 32 and 33 (Fig. 6) are the following: (i) $\mathrm{U} 32$ in panels $\mathrm{A}$ and $\mathrm{B}$ and $\mathrm{A} 38$ in panel A are from E. coli
tRNA $^{\text {Phe }}$ bound to the ribosome A site (PDB ID: 2J00, chain Y); (ii) $\mathrm{C} 32$ in panels $\mathrm{C}$ and $\mathrm{D}$ and $\mathrm{A} 38$ in panel $\mathrm{C}$ are from E. coli tRNA $^{\text {fMet }}$ bound to the ribosome P site (PDB ID: 2J00, chain V); (iii) G38 in panels B and D were modeled by computationally replacing $\mathrm{A} 38$ in the structures shown in panels $\mathrm{A}$ and $\mathrm{B}$, respectively; (iv) $\mathrm{U} 24, \mathrm{U} 69, \mathrm{C} 44$, and $\mathrm{C} 13$ in panels $\mathrm{A}, \mathrm{B}, \mathrm{C}$, and $\mathrm{D}$, respectively, are from the structures of E. coli tRNA ${ }^{\mathrm{fMet}}$ bound to the ribosome P site (PDB ID: 2J00, chain V), yeast tRNA ${ }^{\text {Phe }}$ in the free state (PDB ID: 1EHZ), E. coli tRNA ${ }^{\text {Leu }}$ (PDB ID: 2NQP, chain F) and yeast tRNA $^{\text {Phe }}$ in complex with EF-Tu, PDB ID: 1TTT, chain F) after optimal structural superposition of the base atoms of their respective WC interaction partners (A11, G4, A26, and G22, data not shown) to those of the nucleotide at position 38; (v) U33 in panel E and OMC32, A35, and the phosphate moiety of $\mathrm{A} 36$ in panels $\mathrm{E}$ and $\mathrm{F}$ are from yeast $\mathrm{tRNA}^{\text {Phe }}$ in the free state (PDB ID: 1EHZ); (vi) C33 in panel $F$ was modeled by computationally replacing U33 in the previous structure.

The sequences of tRNA genes were downloaded from two, partially overlapping, databases: (i) "Compilation of tRNA sequences and sequences of tRNA genes" (http://www.uni-bayreuth. de/departments/biochemie/trna/ and http://www.staff.uni-bayreuth. de/ btc914/search/index.html; Sprinzl and Vassilenko 2005), comprising $>3700$ tRNA sequences from different cellular compartments (e.g., mitochondria, cytoplasm, chloroplasts) and from organisms belonging to different kingdoms (e.g., viruses, archaea, eubacteria, single cells and fungi, plants, animals); and (ii) "Compilation of mammalian mitochondrial tRNA genes" (http://mamit-trna. u-strasbg.fr/; Pütz et al. 2007), comprising 3000 tRNA sequences from mammalian mitochondria. The two databases provide complementary information: (i) The Sprinzl and Vassilenko database contains tRNA gene sequences for other cellular compartments as well as mitochondria, and for other species beside mammalian ones; (ii) the Mamit database contains a large number of mt tRNA sequences from mammals that are not included in the previous database. Moreover, since the Sprinzl and Vassilenko database includes polymorphisms, it may contain more than one sequence for the same gene, whereas Mamit contains only one sequence for each of the $22 \mathrm{mt}$ tRNA genes encoded by the genome of each mammalian organism. The sequences downloaded from the two databases were divided into different data sets and analyzed accordingly: all tRNAs from yeast; all tRNAs from all species; all tRNA $^{\text {Ile }}$ from all species; all tRNAs from human; all tRNAs from mammals; and all tRNA ${ }^{\text {Ile }}$ from mammals. For all sets of sequences downloaded from the Sprinzl and Vassilenko database, we analyzed separately tRNAs from mitochondria, cytoplasm of eukaryotic cells, and all cellular compartments (in the case of tRNAs from all species, these include chloroplasts and bacterial or archaeal cells). Detailed results of sequence analyses are reported in Supplemental Tables S4 and S5.

\section{SUPPLEMENTAL MATERIAL}

Supplemental material is available for this article.

\section{ACKNOWLEDGMENTS}

We thank Prof. M. Bolotin-Fukuhara for providing some of the $S$. cerevisiae strains used in this study. This work was supported by grants from Telethon (GGP07164). A.M. was the recipient 
of a Pasteur Institute, Cenci Bolognetti Foundation "Teresa Ariaudo" fellowship.

Received June 23, 2011; accepted July 20, 2011.

\section{REFERENCES}

Agris PF. 1996. The importance of being modified: Roles of modified nucleosides and $\mathrm{Mg}^{2+}$ in RNA structure and function. Prog Nucleic Acid Res Mol Biol 53: 79-129.

Ashraf SS, Ansari G, Guenther R, Sochacka E, Malkiewicz A, Agris PF. 1999. The uridine in "U-turn": Contributions to tRNA-ribosomal binding. RNA 5: 503-511.

Auffinger P, Westhof E. 1999. Singly and bifurcated hydrogen-bonded base-pairs in tRNA anticodon hairpins and ribozymes. J Mol Biol 292: 467-483.

Auffinger P, Westhof E. 2001. An extended structural signature for the tRNA anticodon loop. RNA 7: 334-341.

Baldacci G, Zennaro E. 1982. Mitochondrial transcripts in glucoserepressed cells of Saccharomyces cerevisiae. Eur J Biochem 35: 117123.

Barrientos A. 2003. Yeast models of human mitochondrial diseases. IUBMB Life 55: 83-95.

Basavappa R, Sigler PB. 1991. The $3 \AA$ crystal structure of yeast initiator tRNA: Functional implications in initiator/elongator discrimination. EMBO J 10: 3105-3111.

Berlani ER, Pentella C, Macino G, Tzagoloff A. 1980. Assembly of the mitochondrial membrane system: Isolation of mitochondrial transfer ribonucleic acid mutants and characterization of transfer ribonucleic acid genes of Saccharomyces cerevisiae. J Bacteriol 141: 1986-1997.

Berman HM, Westbrook J, Feng Z, Gilliland G, Bhat TN, Weissig H, Shindyalov IN, Bourne PE. 2000. The Protein Data Bank. Nucleic Acids Res 28: 235-242.

Bolotin-Fukuhara M, Faye G, Fukuhara H. 1977. Temperature sensitive respiratory-deficient mitochondrial mutations: Isolation and genetic mapping. Mol Gen Biol 152: 295-305.

Bonnefoy N, Fox TD. 2007. Directed alteration of Saccharomyces cerevisiae mitochondrial DNA by biolistic transformation and homologous recombination. Methods Mol Biol 372: 153-166.

Brem RB, Yvert G, Clinton R, Kruglyak L. 2002. Genetic dissection of transcriptional regulation in budding yeast. Science 296: 752-755.

De Luca C, Besagni C, Frontali L, Bolotin-Fukuhara M, Francisci S. 2006. Mutations in yeast mt tRNAs: Specific and general suppression by nuclear encoded tRNA interactors. Gene 377: 169-176.

De Luca C, Zhou Y, Montanari A, Morea V, Oliva R, Besagni C, Bolotin-Fukuhara M, Frontali L, Francisci S. 2009. Can yeast be used to study mitochondrial diseases? Biolistic tRNA mutants for the analysis of mechanisms and suppressors. Mitochondrion 9: 408-417.

Feuermann M, Francisci S, Rinaldi T, De Luca C, Rohou H, Frontali L, Bolotin-Fukuhara M. 2003. The yeast counterparts of human 'MELAS' mutations cause mitochondrial dysfunction that can be rescued by overexpression of the mitochondrial translation factor EF-Tu. EMBO Rep 4: 53-58.

Foury F, Rogant T, Lecrenier N, Prunelle B. 1998. The complete sequences of mitochondrial genome of Saccharomyces cerevisiae. FEBS Lett 440: 325-331.

Fox TD, Folley LS, Mulero JJ. 1991. Analysis and manipulation of yeast mitochondrial genes. Methods Enzymol 194: 149-165.

Francisci S, Bohn C, Frontali L, Bolotin-Fukuhara M. 1998. Ts mutations in mitochondrial tRNA genes: Characterization and effects of two point mutations in the mitochondrial gene for tRNAPhe in Saccharomyces cerevisiae. Curr Genet 33: 110-116.

Francisci S, De Luca C, Oliva R, Morea V, Tramontano A, Frontali L. 2005. Aminoacylation and conformational properties of yeast mitochondrial tRNA mutants with respiratory deficiency. RNA 11: 914-927.

Giegé R, Sissler M, Florentz C. 1998. Universal rules and idiosyncratic features in tRNA identity. Nucleic Acids Res 26: 5017-5035.

Herbert CJ, Labouesse M, Dujardin G, Slonimski PP. 1988. The NAM2 proteins from S. cerevisiae and $S$. douglasii are mitochondrial leucyl-tRNA synthetases, and are involved in mRNA splicing. EMBO J 7: 473-483.

Kirino Y, Suzuki T. 2005. Human mitochondrial diseases associated with tRNA wobble modification deficiency. RNA Biol 2: 41-44.

Levinger L, Morl M, Florentz C. 2004. Mitochondrial tRNA 3' end metabolism and human disease. Nucleic Acids Res 32: 5430-5441.

Li R, Guan M. 2010. Human mitochondrial leucyl-tRNA synthetase corrects mitochondrial dysfunctions due to the tRNA ${ }^{\text {Leu(UUR) }}$ A3243G mutation, associated with mitochondrial encephalomyopathy, lactic acidosis and stroke-like symptoms and diabetes. Mol Cell Biol 30: 2147-2154.

Lightowlers RN, Chinnery PF, Turnbull DM, Howell N. 1997. Mammalian mitochondrial genetics: Heredity, heteroplasmy and disease. Trends Genet 13: 450-455.

Limongelli A, Schaefer J, Jackson S, Invernizzi F, Kirino Y, Suzuki T, Reichmann H, Zeviani M. 2004. Variable penetrance of a familial progressive necrotising encephalopathy due to a novel tRNA ${ }^{\text {Ile }}$ homoplasmic mutation in the mitochondrial genome. J Med Genet 41: 342-349.

McFarland R, Clark KM, Morris AA, Taylor RW, Macphail S, Lightowlers RN, Turnbull DM. 2002. Multiple neonatal deaths due to a homoplasmic mitochondrial DNA mutation. Nat Genet 30: $145-146$.

McFarland R, Elson JL, Taylor RW, Howell N, Turnbull DM. 2004. Assigning pathogenicity to mitochondrial tRNA mutations: When "definitely maybe" is not good enough. Trends Genet 20: 591-596.

Montanari A, Besagni C, De Luca C, Morea V, Oliva R, Tramontano A, Bolotin-Fukuhara M, Frontali L, Francisci S. 2008. Yeast as a model of human mitochondrial tRNA base substitutions: Investigation of the molecular basis of respiratory defects. RNA 14: 275-283.

Montanari A, De Luca C, Frontali L, Francisci S. 2010. AminoacyltRNA synthetases are multivalent suppressors of defects due to human equivalent mutations in yeast $\mathrm{mt}$ tRNA genes. $B B A-M C R$ 1803: $1050-1057$.

Moreno-Loshuertos R, Ferrín G, Acín-Pérez R, Gallardo ME, Viscomi C, Pérez-Martos A, Zeviani M, Fernández-Silva P, Enríquez JA. 2011. Evolution meets disease: Penetrance and functional epistasis of mitochondrial tRNA mutations. PLoS Genet 7: e1001379. doi: 10.1371/journal.pgen.1001379.

Mulero JJ, Fox TD. 1993. Alteration of the Saccharomyces cerevisiae COX2 mRNA $5^{\prime}$-untranslated leader by mitochondrial gene replacement and functional interaction with the translational activator protein PET111. Mol Biol Cell 4: 1327-1335.

Olejniczak M, Dale T, Fahlman RP, Uhlenbeck OC. 2005. Idiosyncratic tuning of tRNAs to achieve uniform ribosome binding. Nat Struct Mol Biol 12: 788-793.

Park H, Davison E, King M. 2008. Overexpressed mitochondrial leucyl-tRNA synthetase suppresses the A3243G mutation in the mitochondrial tRNA ${ }^{\mathrm{Leu}(\mathrm{UUR})}$ gene. RNA 14: 2407-2416.

Pütz J, Dupuis B, Sissler M, Florentz C. 2007. Mamit-tRNA, a database of mammalian mitochondrial tRNA primary and secondary structures. RNA 13: 1184-1190.

Rinaldi T, Lande R, Bolotin-Fukuhara M, Frontali L. 1997. Additional copies of the mitochondrial Ef-Tu and aspartyl-tRNA synthetase genes can compensate for a mutation affecting the maturation of the mitochondrial tRNA ${ }^{\text {Asp }}$. Curr Genet 31: 494-496.

Rohou H, Francisci S, Rinaldi T, Frontali L, Bolotin-Fukuhara M. 2001. Reintroduction of a characterized mt tRNA glycine mutation into yeast mitochondria provides a new tool for the study of human neurodegenerative diseases. Yeast 18: 219-227.

Rorbach J, Yusoff AA, Tuppen H, Abg-Kamaludin DP, ChrzanowskaLightowlers ZMA, Taylor RW, Turnbull DM, McFarland R, 


\section{Montanari et al.}

Lightowlers RN. 2008. Overexpression of human mitochondrial valyl tRNA synthetase can partially restore levels of cognate mttRNA $^{\text {Val }}$ carrying the pathogenic C25U mutation. Nucleic Acids Res 36: 3065-3074.

Sambrook J, Fritsch EF, Maniatis T. 1989. Molecular cloning: A laboratory manual. Cold Spring Harbor Laboratory Press, Cold Spring Harbor, NY.

Sasarman F, Antonicka H, Shoubridge EA. 2008. The A3243G tRNA $^{\text {Leu(UUR) }}$ MELAS mutation causes amino acid misincorporation and a combined respiratory chain assembly defect that is partially suppressed by overexpression of the translation elongation factors EFTu and EFG2. Hum Mol Genet 17: 3697-3707.

Schadt EE, Monks SA, Drake TA, Lusis AJ, Che N, Colinayo V, Ruff TG, Milligan SB, Lamb JR, Cavet G, et al. 2003. Genetics of gene expression surveyed in maize, mouse and man. Nature 422: 297-302.

Šponer J, Leszczynski J, Hobza P. 1996. Structures and energies of hydrogen-bonded DNA base pairs. A nonempirical study with inclusion of electron correlation. J Phys Chem 100: 1965-1974.
Sprinzl M, Vassilenko KS. 2005. Compilation of tRNA sequences and sequences of tRNA genes. Nucleic Acids Res 33: D139-D140.

Wallace DC, Fan WW. 2009. The pathophysiology of mitochondrial disease as modeled in the mouse. Genes Dev 23: 1714-1736.

Wilson FH, Hariri A, Farhi A, Zhao H, Petersen KF, Toka HR, Nelson-Williams C, Raja KM, Kashgarian M, Shulman GI, et al. 2004. A cluster of metabolic defects caused by mutation in a mitochondrial tRNA. Science 306: 1190-1194.

Wittenhagen LM, Kelley SO. 2003. Impact of disease-related mitochondrial mutations on tRNA structure and function. Trends Biochem Sci 28: 605-611.

Yvert G, Brem RB, Whittle J, Akey JM, Foss E, Smith EN, Mackelprang R, Kruglyak L. 2003. Trans-acting regulatory variation in Saccharomyces cerevisiae and the role of transcription factors. Nat Genet 35: 57-64.

Zennaro E, Francisci S, Ragnini A, Frontali L, Bolotin-Fukuhara M. 1989. A point mutation in a mitochondrial tRNA gene abolishes its 3'end processing. Nucleic Acids Res 17: 5751-5764. 

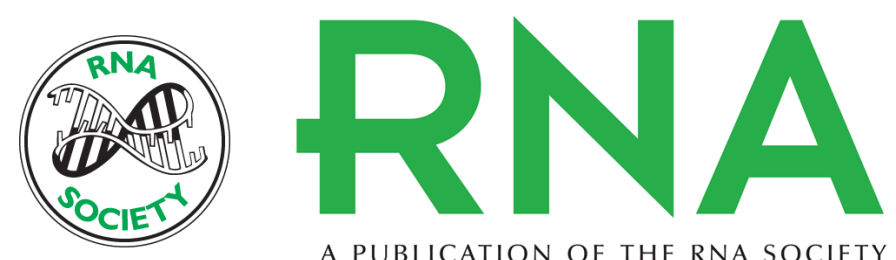

A PUBLICATION OF THE RNA SOCIETY

\section{Structural and functional role of bases 32 and 33 in the anticodon loop of yeast mitochondrial tRNA}

Arianna Montanari, Cristina De Luca, Patrizio Di Micco, et al.

RNA 2011 17: 1983-1996 originally published online September 13, 2011

Access the most recent version at doi:10.1261/rna.2878711

\section{Supplemental http://rnajournal.cshlp.org/content/suppl/2011/08/03/rna.2878711.DC1 \\ Material}

References This article cites 47 articles, 12 of which can be accessed free at: http://rnajournal.cshlp.org/content/17/11/1983.full.html\#ref-list-1

\section{License}

Email Alerting Receive free email alerts when new articles cite this article - sign up in the box at the Service top right corner of the article or click here.

\section{IIIII!' Providing Precise Solutions for your research.}

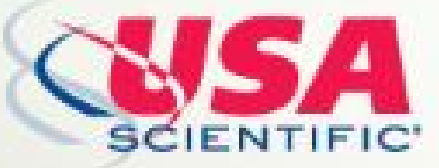

To subscribe to RNA go to:

http://rnajournal.cshlp.org/subscriptions 